years, then declined as range conditions deteriorated. Mr Blair Rains's examples came from Botswana and the Gambia, where control against rinderpest and other bovine diseases has been startlingly successful. Although cattle marketing is developing, large herds are still kept for traditional purposes, water supplies are no longer limiting, and inability to control stocking levels has resulted in either shrub encroachment or in the nearly complete destruction of vegetation over very large areas around water points.

Dr D. C. Dawkins (Commonwealth Forestry Institute, Oxford) spoke on the effects of forest management, with operations which range in scale from merely weeding out hollow trees, resulting in the loss of holes for tree dwelling fauna, to total clearing and replacement by forest monocultures, often of exotic species. His colleague Mr P. J. Wood then discussed ecological anxieties arising from these monocultures which are now replacing forest and grassland in many tropical lands; in the East African Highlands alone some 15,000 hectares of conifers are being planted each year. The long-term effects of these monocultures have to date been little studied, but the evidence available suggests that the resulting erosion and alteration of soil profiles present greater dangers than the introductions of pests and pathogens, or than changes in water balance or soil microflora. The "ecological spillover" effects are most in need of study. The ensuing discussion brought out the problems of ensuring that areas of indigenous forest are preserved for conservation purposes in countries which are now independent.

The unprecedented pressures of affluence and cheap travel on the limited tropical littoral resources of West Indian islands were treated in stimulating and well illustrated contributions by $\mathrm{Mr}$ M. A. Brunt (Land Resources Division, Overseas Development Administration) and by Dr M. E. C. Giglioli (Mosquito Research and Control Unit, Cayman Islands). In Jamaica, the replacement of mangrove swamps by man-made beaches has provided suitable breeding places for biting sand flies (Culicoides), with the result that there have been local infestations of these insects. In the Cayman Islands, mangrove swamps have been flooded to control the biting mosquito Aëdes taeniorhynchus, and cleared and filled with marl for housing development. The cleared areas have remained bare of vegetation, leaving the land exposed to damage from hurricanes which frequent this area. $\mathrm{Dr}$ Giglioli described aerial spraying operations, and stressed the importance of inviting local people to share in control operations to ensure the acceptance of the necessary measures by the local community.
OCEANIC RIDGES

\section{Problems with Chemistry}

from our Geomagnetism Correspondent

THE general hypothesis of seafloor spreading envisages the upwelling of peridotitic mantle material beneath midoceanic ridges which are known to be regions of relatively high heat flow. Partial melting of the material then takes place producing basaltic liquids which are injected into comparatively narrow regions near the ridge axis. The existence of high thermal régimes beneath ridge axes implies that beneath ridges the silicate melts are generated at shallower depths, and thus lower pressures, than would normally be the case. Because the compositions of liquids arising from partial melting of mantle material are strongly dependent upon the pressure under which such liquids are produced, the prediction is then made that the compositions of basalts injected at ridge axes should be significantly different from that of volcanic rocks produced well away from ridges. In particular, if the simple theory is valid, ridge basalts should tend to be tholeiitic whereas non-ridge basalts would be expected to be more alkaline.

But these cherished assumptions have taken a sharp knock with the publication by Bonatti and Fisher (Earth Planet.
Sci. Lett., 11, 307 ; 1971) of compositional data for basalts from the East Pacific Rise and other Pacific sites removed from the rise. Most of the rise rocks in question were from a basalt pavement which outcrops along the axis of the rise between $14^{\circ} \mathrm{S}$ and $6^{\circ} \mathrm{S}$ though two others were obtained from $18^{\circ} \mathrm{S}$ and near to the equator, respectively. All were less than one million years old according to radiometric or fission track dating. The non-rise samples were collected from five seamounts lying between 450 and $1,500 \mathrm{~km}$ from the rise axis and all were less than five million years old, again according to radiometric or fission track dating. It is possible, of course, that the seamount samples were emplaced at the rise axis and subsequently spread away to their present positions and if this were so-and assuming a reasonable spreading rate of $4 \mathrm{~cm}$ a year-their ages would lie in the approximate range 11-35 million years. The measured ages rule this out, however, and suggest that, on the contrary, the seamounts were formed more or less in their present positions. The contrast between rise and non-rise basalts is thus valid.

The surprise comes from comparisons of normative compositions and the degree of silica saturation for the rise and non-rise basalts. When normative compositions are plotted on the Q-Di-

\title{
Similarities between Pulsar Models
}

THE two widely favoured rotating neutron star models for pulsars differ less widely than is sometimes believed, according to an article by $\mathrm{L}$. Mestel, of the University of Manchester, in next Monday's Nature Physical Science. These two models are the axisymmetric model of Goldreich and Julian (the "non-oblique rotator") and the oblique rotator of Pacini and Ostriker and Gunn.

Goldreich and Julian found that for their model, with its relatively dense magnetosphere, the vacuum electric field $\left(\mathbf{D}=\varepsilon_{0} \mathbf{E}\right)$ just outside the surface of the star has a component along the magnetic field which produces electrical forces on the charges in the surface layer which are much greater than the gravitational forces. This results in a motion of electrons out of the star, so that the steady state of the model includes a charged magnetosphere. Currents produced by the bulk motions of the charge distribution determine a field structure typified by a corotating region of field lines which close within the light cylinder and a wind zone defined by the lines of force which cross the light cylinder, and allow charge to flow outwards. But even at points well within the light cylinder the magnetosphere is relativistic, in the sense that the magnetic energy density is much greater than the mass energy density.

When the angle between the magnetic and rotation axes is non-zero, the electromagnetic and plasma fields around the pulsar are non-steady. But it seems inevitable that charges will still be removed from the star, as in the Goldreich and Julian model. The details of the magnetic field structure are less easy to determine than for the axisymmetric case, but Mestel puts forward a qualitatively similar view in which, as for the Goldreich and Julian model, the field obeys the curl free (dipole) approximation to a good accuracy at points well within the light cylinder. For both models, provided that the hydromagnetic condition

$$
\mathbf{E}+\frac{\mathbf{V} \times \mathbf{B}}{c}=\mathbf{0}
$$

is obeyed, energy outflow occurs only along field lines which cross the speed of light cylinder, and classic magnetic dipole radiation from the curl free zone is suppressed. For a poloidal field the torque will vary as $(\Omega a / c)^{2 n-3}$. For a dipole $n=3$, but the work of Pacini and Rees (Nature, 226, 622; 1970) suggests that in the Crab pulsar $n \sim 2.6$, which tends to confirm the non-dipole nature of the energy loss mechanism. 